\title{
Abordagem de crise miastênica infanto-juvenil: relato de caso
}

\author{
Approach to juvenile myasthenic crisis: case report \\ Abordaje de la crisis miasténica juvenil: reporte de caso
}

Recebido: 21/09/2021 | Revisado: 27/09/2021 | Aceito: 28/09/2021 | Publicado: 01/10/2021

\author{
Murilo Henrique Godinho Roque \\ ORCID: https://orcid.org/0000-0001-9043-7043 \\ Centro Universitário de Patos de Minas, Brasil \\ E-mail: murilogodinho@unipam.edu.br \\ Bárbara Queiroz de Figueiredo \\ ORCID: https://orcid.org/0000-0003-1630-4597 \\ Centro Universitário de Patos de Minas, Brasil \\ E-mail: barbarafigueiredo@unipam.edu.br \\ Iuri Pimenta Oliveira \\ ORCID: https://orcid.org/0000-0002-9004-1513 \\ Centro Universitário de Patos de Minas, Brasil \\ E-mail: iuripimenta@unipam.edu.br \\ Mychell Flávio Aparecido Rodrigues de Sousa \\ ORCID: https://orcid.org/0000-0003-1132-2761 \\ Centro Universitário de Patos de Minas, Brasil \\ E-mail: mychel.flavio@ hotmail.com \\ Otávio Augusto de Freitas \\ ORCID: https://orcid.org/0000-0003-2279-6233 \\ Hospital Regional Antônio Dias, Brasil \\ E-mail: otavioauf@yahoo.com.br \\ Vítor Augusto Ferreira Braga \\ ORCID: https://orcid.org/0000-0002-3927-096X \\ Centro Universitário de Patos de Minas, Brasil \\ E-mail: vitoraugustofb@gmail.com \\ Rúbia Carla Oliveira \\ ORCID: https://orcid.org/0000-0002-7162-3497 \\ Centro Universitário de Patos de Minas, Brasil \\ E-mail: rubiacoliveira@unipam.edu.br
}

\begin{abstract}
Resumo
Introdução: a Miastenia gravis (MG) é uma doença autoimune que afeta a junção neuromuscular, a produção de anticorpos juntamente com o sistema complemento, atua contra os receptores nicotínicos da acetilcolina (AChR), localizados na membrana pós sináptica, resultando em significativo comprometimento da transmissão neuromuscular. O quadro clínico clássico se apresenta por fraqueza flutuante e fatigabilidade da musculatura ocular, facial, bulbar ou de membros. A crise miastênica é definida como insuficiência respiratória associada a fraqueza muscular grave, é uma complicação potencialmente fatal que ocorre em aproximadamente 15\%-20\% dos pacientes com MG. Objetivo: demonstrar a abordagem na crise miastênica, e dificuldade diagnóstica da crise miastênica, melhorando o prognostico dos jovens acometidos, identificando fatores que possam vir a desencadear o início dos sintomas, podendo assim qualificar e antecipar o diagnóstico da crise e tratamento, no intuito de que não ocorram procedimentos inadequados aos portadores da doença. Metodologia: trata-se de um estudo de caso clínico com perspectiva qualitativa e descritiva, que consiste em uma pesquisa em que, em geral, ocorre com coleta direta de dados, cujo o pesquisador é o instrumento indispensável. Relato de caso: Trata-se de paciente de 11 anos, sexo feminino, negra, com diagnóstico prévio de Miastenia Gravis há 6 meses, com quadro de fraqueza muscular proximal difusa, admitida de vaga zero na unidade de pronto atendimento do hospital em questão com quadro de falência da musculatura respiratória aguda, sedação superficial, administração de fentanil e midazolan, e foi encaminhada a unidade de terapia intensiva, mantendo-se em ventilação mecânica. Conclusão: frente ao caso relatado, nota-se a importância de um diagnóstico preciso, atendimento rápido e eficaz, mantendo sempre em vista a estabilização do quadro de crise, suporte ventilatório, e tratamento farmacológico especializado, usando em vista a imunoglobulina que apresentou boa eficácia no tratamento, e dispondo de equipe multidisciplinar em unidade de terapia intensiva, constituída pelo fisioterapeuta respiratório principalmente em quadros respiratórios, para ofertarmos o suporte adequado ao tratamento de doenças raras e melhor manejo da ventilação no caso de pacientes infanto juvenis com miastenia gravis.
\end{abstract}

Palavras-chave: Miastenia; Crise Miastênica; Criança; Conduta. 


\begin{abstract}
Introduction: Myasthenia gravis (MG) is an autoimmune disease that affects the neuromuscular junction, the production of antibodies together with the complement system, acts against nicotinic acetylcholine receptors (AChR), located in the postsynaptic membrane, resulting in significant impairment of the neuromuscular transmission. The classic clinical picture presents with fluctuating weakness and fatigability of the ocular, facial, bulbar or limb muscles. Myasthenic crisis, defined as respiratory failure associated with severe muscle weakness, is a potentially fatal complication that occurs in approximately $15 \%-20 \%$ of patients with MG. Objective: to demonstrate the approach to the myasthenic crisis, and the difficulty in diagnosing the myasthenic crisis, improving the prognosis of affected young people, identifying factors that may trigger the onset of symptoms, thus being able to qualify and anticipate the diagnosis of the crisis and treatment, in order to that inadequate procedures do not occur to patients with the disease. Methodology: this is a clinical case study with a qualitative and descriptive perspective, which consists of a research that, in general, takes place with direct data collection, in which the researcher is the indispensable instrument. Case report: This is an 11-year-old female patient, black, with a previous diagnosis of Myasthenia Gravis 6 months ago, with diffuse proximal muscle weakness, admitted for zero vacancy in the emergency care unit of the hospital in question with acute respiratory muscle failure, superficial sedation, administration of fentanyl and midazolam, and she was referred to the intensive care unit, maintaining mechanical ventilation. Conclusion: in view of the case reported, the importance of an accurate diagnosis, quick and effective care is noted, always keeping in mind the stabilization of the crisis, ventilatory support, and specialized pharmacological treatment, using immunoglobulin that showed good efficacy. in the treatment, and having a multidisciplinary team in an intensive care unit, consisting of a respiratory physiotherapist, mainly in respiratory conditions, to offer adequate support for the treatment of rare diseases and better ventilation management in the case of juvenile patients with myasthenia gravis.
\end{abstract}

Keywords: Myasthenia; Myasthenic Crisis; Kid; Conduct.

\begin{abstract}
Resumen
Introducción: La miastenia gravis (MG) es una enfermedad autoinmune que afecta la unión neuromuscular, la producción de anticuerpos junto con el sistema del complemento, actúa contra los receptores nicotínicos de acetilcolina (AChR), localizados en la membrana postsináptica, resultando en un deterioro significativo de la transmisión neuromuscular. . El cuadro clínico clásico se presenta con debilidad fluctuante y fatiga de los músculos oculares, faciales, bulbares o de las extremidades. La crisis miasténica, definida como insuficiencia respiratoria asociada con debilidad muscular severa, es una complicación potencialmente fatal que ocurre en aproximadamente el 15\% -20\% de los pacientes con MG. Objetivo: demostrar el abordaje de la crisis miasténica, y la dificultad en el diagnóstico de la crisis miasténica, mejorando el pronóstico de los jóvenes afectados, identificando factores que pueden desencadenar la aparición de síntomas, pudiendo calificar y anticipar el diagnóstico de la crisis. y tratamiento, para que no se produzcan procedimientos inadecuados a los pacientes con la enfermedad. Metodología: se trata de un estudio de caso clínico con perspectiva cualitativa y descriptiva, que consiste en una investigación que, en general, se realiza con recolección directa de datos, en la que el investigador es el instrumento indispensable. Caso clínico: Se trata de una paciente de 11 años, de raza negra, con diagnóstico previo de Miastenia Gravis hace 6 meses, con debilidad muscular proximal difusa, ingresada por vacante cero en la unidad de urgencias del hospital de que se trate por cuadro respiratorio agudo. insuficiencia muscular, sedación superficial, administración de fentanilo y midazolam, y fue remitida a la unidad de cuidados intensivos, manteniendo ventilación mecánica. Conclusión: a la vista del caso reportado se destaca la importancia de un diagnóstico certero, atención rápida y eficaz, siempre teniendo en cuenta la estabilización de la crisis, el soporte ventilatorio y el tratamiento farmacológico especializado, utilizando inmunoglobulina que mostró buena eficacia. tratamiento, y contar con un equipo multidisciplinar en una unidad de cuidados intensivos, compuesto por un fisioterapeuta respiratorio, principalmente en afecciones respiratorias, para ofrecer un soporte adecuado para el tratamiento de enfermedades raras y un mejor manejo de la ventilación en el caso de pacientes juveniles con miastenia gravis.
\end{abstract}

Palabras clave: Miastenia; Crisis Miasténica; Niño; Conducta.

\title{
1. Introdução
}

A Miastenia gravis (MG) é uma doença autoimune que afeta a junção neuromuscular (Hughes et al. 2006), a produção de anticorpos juntamente com o sistema complemento, atua contra os receptores nicotínicos da acetilcolina (AChR), localizados na membrana pós sináptica, resultando em significativo comprometimento da transmissão neuromuscular (Kauling et al. 2011). O quadro clínico clássico se apresenta por fraqueza flutuante e fatigabilidade da musculatura ocular, facial, bulbar ou de membros (Marques et al. 2020). Um quadro de miastenia gravis juvenil (MGJ) inclui os indivíduos com até 19 anos e ainda é subdividida, de acordo com idade em que se apresenta o primeiro sintoma, em pré-púbere (antes dos 12 anos) e póspúbere (após os 12 anos), além de ser reconhecida como um distúrbio raro na população pediátrica (Rodrigues et al. 2016). 
A crise miastênica é definida como insuficiência respiratória associada a fraqueza muscular grave, é uma complicação potencialmente fatal que ocorre em aproximadamente 15\%-20\% dos pacientes com MG (Costa, 2016). A mortalidade nesses casos caiu de 40\% no início da década de 1960 para apenas 4\% a partir de meados da década de 1970, devido em grande parte à melhora dos cuidados respiratórios e das unidades de terapia intensiva e, em menor parcela, devido ao amplo uso de imunoterapias como plasmaférese e imunoglobulina humana. (Ministério da Saúde, 2015).

O tratamento da MG objetiva o controle dos sintomas motores característicos, a diminuição das exacerbações, o aumento do período em remissão e tratamento das crises miastênicas (Keesey, 2002). O tratamento medicamentoso pode ser direcionado para alivio de sintomas e para reduzir a produção dos autoanticorpos. Para alívio dos sintomas, os medicamentos visam aumentar a ação da acetilcolina na fenda sináptica, possibilitando o retorno da força muscular (Lopez et al. 2008). Para redução da produção de anticorpos, uma vertente do tratamento é a imunomodulação com uso de imunossupressores capazes de reduzir essa resposta. Os medicamentos mais utilizados são prednisona, piridostigmina, ciclosporina, azatioprina, ciclofosfamida e a imunoglobulina humana (Marques et al. 2020). Sob essa perspectiva, o objetivo deste estudo visa demonstrar a abordagem na crise miastênica, e dificuldade diagnóstica da MGJ, melhorando o prognostico dos jovens acometidos, identificando fatores que possam vir a desencadear o início dos sintomas, podendo assim qualificar e antecipar o diagnóstico da crise e tratamento, no intuito de que não ocorram procedimentos inadequados aos portadores da doença.

\section{Metodologia}

Este artigo trata-se de um estudo de caso clínico com perspectiva qualitativa e descritiva, que consiste em uma pesquisa em que, em geral, ocorre com coleta direta de dados, cujo o pesquisador é o instrumento indispensável. O presente estudo contou com o aporte de trabalhos acadêmicos entre os anos de 2002 e 2020. Por meio do acesso às principais bases de dados, a saber: dados National Library of Medicine (PubMed MEDLINE), Scientific Electronic Library Online (Scielo), Cochrane Database of Systematic Reviews (CDSR), Google Scholar, Biblioteca Virtual em Saúde (BVS) e EBSCO Information Services, no período compreendido entre novembro e dezembro de 2020. O Termo de Consentimento Livre e Assistido (TCLE) foi assinado pelo paciente para coleta de dados neste estudo.

\section{Relato de Caso}

Trata-se de paciente de 11 anos, sexo feminino, negra, com diagnóstico prévio de Miastenia Gravis há 6 meses, com quadro de fraqueza muscular proximal difusa, sob uso de Mestinon 300mg por dia, Meticortem 40mg por dia, em acompanhamento com neurologista, admitida de vaga zero na unidade de pronto atendimento do hospital em questão com quadro de falência da musculatura respiratória aguda, sedação superficial, administração de fentanil e midazolan, e foi encaminhada a unidade de terapia intensiva, mantendo-se em ventilação mecânica. Apresentou ptose palpebral a esquerda, mobilidade muscular reduzida em membros inferiores e superiores. Foram realizados exames laboratoriais, conforme ilustrado pelo Quadro 1. 
Quadro 1: Exames laboratoriais realizados.

\begin{tabular}{|c|c|c|}
\hline Elementos dosados & Dosagens & Valores de referência \\
\hline Hemoglobina & $12,6 \mathrm{~g} / \mathrm{dL}$ & $13,5 \mathrm{a} 15,5 \mathrm{~g} / \mathrm{dL}$ \\
\hline Leucócitos & $22.630 \mu \mathrm{L}$ & $3.600 \mathrm{a} 11.000 / \mu \mathrm{L}$ \\
\hline Plaquetas & $200.000 \mathrm{~mm}^{3}$ & $140000 \mathrm{a} 450000 \mu \mathrm{L}$ \\
\hline Globulina & $2,8 \mathrm{~g} / \mathrm{dL}$ & $6 \mathrm{a} 8 \mathrm{~g} / \mathrm{dL}$ \\
\hline Neutrófilos Bastonetes & $2,27 \mu \mathrm{L}$ & $0 \mathrm{a} 800 / \mu \mathrm{L}$ \\
\hline Bilirrubina & $2,26 \mathrm{mg} / \mathrm{dL}$ & Até $1,2 \mathrm{mg} / \mathrm{dL}$ \\
\hline Anticorpo anti-receptor de acetilcolina & $0,33 \mathrm{nmol} / \mathrm{L}$ & Negativo: Inferior a $0,25 \mathrm{nmol} / \mathrm{L}$ \\
\hline $\mathrm{PH}$ & 7,29 & $7.35-7.45$ \\
\hline $\mathrm{pCO}^{2}$ & $53 \mathrm{mmHg}$ & $35-45 \mathrm{mmHg}$. \\
\hline $\mathrm{pO}^{2}$ & $77 \mathrm{mmHg}$ & $80-100 \mathrm{mmHg}$. \\
\hline $\mathrm{BCO}^{3}$ & $25 \mathrm{mEq} / \mathrm{L}$ & $22-26 \mathrm{mEq} / \mathrm{L}$ \\
\hline
\end{tabular}

Fonte: Achados laboratoriais da paciente (2021).

Devido diagnóstico de crise miastênica, foi suspendida a terapia anticolinesterásica básica, descartando síndrome colinérgica, iniciado tratamento com imunoglobulina humana $100 \mathrm{mg} / \mathrm{ml}$ por 5 dias, antibioticoterapia profilática à pneumonia bacteriana por broncoaspiração, associado fisioterapia respiratória e motora, vigilância infecciosa, e descartado hipótese de infecção por SARS-CoV-2. Com o início imediato do tratamento da crise miastênica, a paciente evoluiu significativamente bem, apresentando melhora no padrão de força muscular após 5 dias e continuou em ventilação mecânica em modo de pressão controlada, onde segue o drive respiratório da mesma, consciente e contactuante, responsiva a comandos, caminhar de aproximadamente 10 metros e mantendo padrão respiratório em ventilação mecânica via tubo oro traqueal. Apresentou melhora do prognóstico, e foi realizado protocolo de extubação, sendo favorável ao procedimento.

À extubação realizada, apresentou falência respiratória e broncoaspiração, sendo procedido intubação orotraqueal, em 1 hora após extubação. Sem prognóstico de sustentação sem o suporte ventilatório, procedeu-se com realização de traqueostomia, mantendo, assim, sob ventilação mecânica no modo de pressão de suporte. Após dois dias feita a decanulação, a paciente manteve em ar ambiente, com bom padrão respiratório, sem alterações laboratoriais, mantendo bom quadro clínico e recebeu alta com orientação de acompanhamento neurológico e tratamento da patologia em questão.

\section{Discussão}

É importante que crise miastênica seja diferenciada da crise colinérgica (Scherer et al. 2005), situação de quadro clínico muito semelhante à anterior, que ocorre devido a doses muito altas de anticolinesterásico, se assemelhando a intoxicação por organofosforado, que pode ocupar o mesmo receptor que a acetilcolina, reduzindo a transmissão neuromuscular (Margotto et al. 2012). Tendo em vista também o aumento dos quadros de insuficiência respiratória aguda por infecção pelo coronavírus, onde apresenta sintomas agudos, característicos que é facilmente uma hipótese diagnóstica (Meriggioli et al. 2009).

Em alguns pacientes o diagnóstico se torna difícil principalmente aqueles que cursam com quadro de fraqueza focal de determinados grupos musculares, como os músculos respiratórios (Lorenzoni et al. 2011), além de existir um longo período entre a instalação dos sintomas e o diagnóstico (Santos et al. 2014). A dificuldade no diagnóstico ocorre pelo fato da MG ser uma doença relativamente rara, e apresentar sintomas semelhantes aos de doenças mais comuns, como acidente vascular encefálico, doença do neurônio motor ou histeria podem ser diagnosticados erroneamente (Souza et al. 2014). Além disso, a característica flutuante da doença também pode gerar confusão e atraso no diagnóstico acarretando em um tratamento inadequado até mesmo iatrogênico (Castello-Branco, 2011). 


\section{Conclusão}

Frente ao caso relatado, nota-se a importância de um diagnóstico preciso, atendimento rápido e eficaz, mantendo sempre em vista a estabilização do quadro de crise, suporte ventilatório, e tratamento farmacológico especializado, usando em vista a imunoglobulina que apresentou boa eficácia no tratamento, e dispondo de equipe multidisciplinar em unidade de terapia intensiva, constituída pelo fisioterapeuta respiratório principalmente em quadros respiratórios, para ofertarmos o suporte adequado ao tratamento de doenças raras e melhor manejo da ventilação no caso de pacientes infanto juvenis com miastenia gravis.

\section{Referências}

Castello-Branco, A. C. S., et al. (2011). Atualizações e Perspectivas na Miastenia gravis. Revista Brasileira de Ciências da Saúde, 15 (4), $493-506$.

Costa, H. C. R. A. (2016). Miastenia Gravis: aspectos epidemiológicos e evidências sanitárias no Brasil, no período de 2009 a 2013.2016 .32 f., il. Trabalho de conclusão de curso (Bacharelado em Gestão em Saúde Coletiva) - Universidade de Brasília, Brasília, 2016.

Hughes, B. W., et al. (2006). Arquitetura molecular da junção neuromuscular. Muscle Nerve, 33 (8), 445-461.

Kauling, A. L., et al. (2011). Miastenia Gravis: Relato de Dois Casos e Revisão da Literatura. Revista Brasileira de Anestesiologia, 61 (6), $748-763$.

Keesey, J. C. (2002). “Crise” na miastenia gravis: uma perspectiva histórica. Muscle Nerve, 26 (6), 1-3.

Lopez, M. J., et al. (2008). Miastenia grave en la adolescencia: A propósito de un caso. Rev Cubana Med Gen Integr, Ciudad de La Habana, 24 (2), 4-9.

Lorenzoni, P. J., et al. (2011). Myasthenia gravis complicated with cryptococcal meningitis after thymectomy and long-term immunosuppressive therapy. Arquivos de neuro-psiquiatria, 69 (2), 410-411.

Margotto, P. R., et al. (2012). Miastenia grave neonatal: relato de caso. Brasília Med., 49 (1), 66-70.

Marques, L. G., et al. (2020). Miastenia gravis juvenil: Dificuldade diagnóstica na pediatria. Residência Pediátrica, 10 (1), 3-10.

Meriggioli, M. N., et al. (2009). Autoimmune myasthenia gravis: emerging clinical and biological heterogeneity. Lancet Neurol, 8 (5), $475-490$.

Ministério da Saúde (2015). Protocolo clínico e Diretrizes Terapêuticas- Miastenia Gravis. http://portalarquivos.saude.go v.br/images/p df/2015/novem bro/2 6/PT-SAS-N---1-169-Miastenia-Gravis-ATUALIZADO-19-11-2015.pdf

Rodrigues, M. M. (2016). Tratado de Neurologia Infantil, Editora Atheneu, 1-1264.

Santos, B. F., et al. (2014). Beneficios de un programa de ejercicio multicomponente de baja intensidad y corta duración en la miastenia gravis. A propósito de un caso. Revista Andaluza de Medicina del Deporte, 7 (4), 178-181.

Scherer, K. et al. (2005). Does this patient have myasthenia gravis? JAMA, 293 (15), 1906-14

Souza, L. M., et al. (2014). Grau de dependência para desenvolver atividades instrumentais da vida diária em adultos com Miastenia Grave. Revista Ampliar, 1 (1), 3-11. 